\title{
Depolarized Rayleigh Scattering and Stereoregularity of Polystyrene Chains.* I. Experimental
}

\author{
Georges Fourche and Marie-Thérèse JACQ \\ Centre de Recherches Paul Pascal, Domaine Universitaire \\ 33, Talence, France.
}

(Received March 27, 1972)

\begin{abstract}
The study of the average molecular optical anisotropy $\left\langle\gamma^{2}\right\rangle$ of atactic and isotactic polystyrene chains together with their oligomers has been carried out by means of depolarized Rayleigh scattering.

In the case of atactic compounds a systematic study of molecular optical anisotropy has also been made as a function of the degree of polymerization, polydispersity, and solvent.

The comparison of the experimental optical anisotropies of atactic and isotactic structures reveals a considerable difference, approximately 10 fold, thus pointing out the specific importance of the structural information that we can hope to obtain from the optical anisotropy.

KEY WORDS Optical Anisotropy / Rayleigh Scattering / Stereoregularity / Polystyrene / Diphenylpentane /
\end{abstract}

The results presented in the last few years by Tsvetkov, et al., ${ }^{1}$ on the stereoregularity of isotactic, syndiotactic, and atactic polymers have pointed out the high sensitivity of the optical anisotropy of the "statistical segment" (deduced from flow birefringence or photoelasticity measurements) to the degree of tacticity of the molecule. The problem of stereoregularity for molecular chains in solution presents, from a fundamental point of view, an essential link in the understanding of important biological mechanisms, when it is realized that the chains involved (nucleic acids, proteins) always have a stereoregular structure.

Furthermore, with the exception of already old studies carried out by Benoit, ${ }^{2}$ Horn, ${ }^{3}$ and Weill, ${ }^{4}$ little theoretical and experimental work has been done on the molecular optical anisotropy of polymers. Indeed, there exists no recent study of optical anisotropy of chain molecules as a function of their structure, degree of polymerization, and the solvent used.

Therefore we have undertaken the study by

* Part of this work was presented (G-F) at the 9th microsymposium on "Thermodynamics of interactions in polymer solutions,"' Prague, Sept. 6-9, 1971. depolarized Rayleigh scattering (a simple and direct technique) of the molecular optical anisotropy of atactic and isotactic polystyrene (PS) chains in solution.

Since the success in synthetizing high crystalline polymers with high stereoregularity, the physico-chemical properties of these molecules have been subjected to numerous investigations. The work by Utiyama ${ }^{20}$ in particular first indicated the importance of anisotropic Rayleigh scattering in the study of stereoregular polymers and stimulated the more extensive studies.

The hindrance to internal rotation around each $\mathrm{C}-\mathrm{C}$ bond of the main chain must certainly be affected by the stereochemical configuration of asymmetric carbons. This is why several authors have tried to analyze the effect of this local structural arrangement (microtacticity) on the short range interactions in the chain, that is to say on molecular properties such as mean dimensions and dipole moments. The results obtained are generally disappointing. Indeed, in almost all cases, with the exception of poly(methyl methacrylate) (PMM) in acetone ${ }^{5}$ the sizes of atactic and isotactic polymers have been found to be approximately identical. It must be noticed that all these measurements 
have been carried out in nonideal solutions in which the extension of the macromolecule is determined both by short range (rotational isomerism) and long range (excluded volume) interactions in the chain. Under these conditions the observed identity between atactic and isotactic chain dimensions in solution may only be the result of a compensation effect from long range interactions.

Thus, fairly recently, some of these experiments have been repeated in theta solvents in order to obtain the unperturbed dimensions of these polymers. The results show that the dimensions of isotactic chains are in general 10 to $15 \%$ larger, at most, than the corresponding atactic chains. The same observations are generally applicable to the dipole moment. The effects shown above are within the range of experimental inaccuracy and none of the classical techniques of physical chemistry (viscosity, light scattering, and osmotic pressure) could pick out sharp differences between homologous atactic and isotactic polymers in solution.

In this work we are reporting the first results obtained by depolarized Rayleigh scattering on the average molecular optical anisotropy $\left\langle\gamma^{2}\right\rangle$ of atactic and isotactic polystyrene chains in various solvents.

Since the experiment has revealed a considerable difference between molecular optical anisotropies of atactic and isotactic structures, we have tried to interpret (see part II of this series) these observations in terms of short range interactions in the molecule and to determine the preferred conformations of these chains in solution.

Moreover, in order to examine the possibility of extending the study of molecular optical anisotropy to polymer chains having a large degree of polymerization, we have undertaken, for atactic compounds, a systematic study of a series of fractions with molecular weights ranging from 560 to 498000 .

\section{EXPERIMENTAL TECHNIQUE}

A detailed description of the experimental technique used can be found in previous work..$^{6,7}$ We shall only give a review of the theory necessary to an understanding of this study.

\section{Scattered Depolarized Intensity}

When a beam of parallel monochromatic light, linearly polarized, impinges on anisotropic molecules, they scatter in the $O X$ direction (see Figure 1) a light wave which can be decomposed into two perpendicular vibrations $I$ and $i$ having the same frequency as the exciting wave. The optical anisotropy of the molecules appears in the direction of observation perpendicular to the incident beam direction on the basis of depolarized intensity $i$ which can be isolated by a Glazebrook prism $G$, and which is parallel to the incident beam direction. It can be shown ${ }^{8}$ that the scattered depolarized intensity is directly* related to the optical anisotropy $\gamma^{2}$ of molecules, which is defined by the following expression

$$
\begin{aligned}
\gamma^{2}= & \frac{1}{2}\left[\left(\alpha_{X X}-\alpha_{Y Y}\right)^{2}+\left(\alpha_{Y Y}-\alpha_{Z Z}\right)^{2}+\left(\alpha_{Z Z}-\alpha_{X X}\right)^{2}\right] \\
& +3\left[\alpha_{X Y}^{2}+\alpha_{Y Z}^{2}+\alpha_{Z X}^{2}\right]
\end{aligned}
$$

where $\alpha_{X X}, \alpha_{Y Y}$, etc., are the components of the molecular polarizability tensor $\boldsymbol{\alpha}$, with respect to fixed molecular cartesian axes $O X Y Z$. In the case of molecular chains having, from the optical standpoint, bonds of axial symmetry, it is more convenient to write the molecular optical anisotropy in the general form,

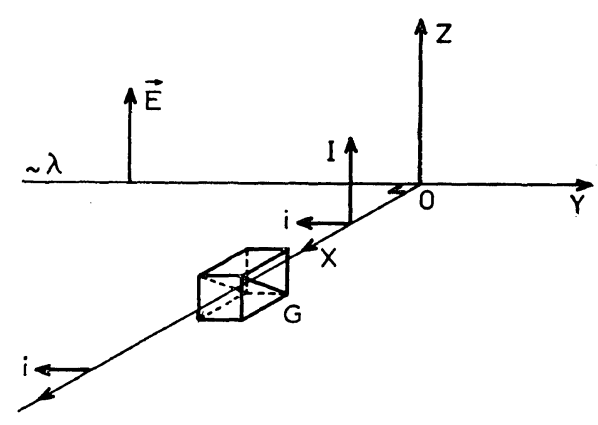

Figure 1. Scattered depolarized intensity.

* The scattered light also may be due to phenomena other than inherent optical anisotropy of the molecule, such as molecular collisions ${ }^{30}$ or radial correlations ${ }^{31}$ in the condensed medium. However, in the case of highly anisotropic polymer chains, in general, the scattered intensity due to collision induced anisotropy or radial correlations can be neglected to a good approximation. ${ }^{31,32}$ 


$$
\gamma^{2}=\frac{1}{2} \sum_{j, k} \gamma_{j} \gamma_{k}\left(3 \cos ^{2} \theta_{j k}-1\right)
$$

where $\gamma_{j}$ is the optical anisotropy of bond $j$, and $\theta_{j k}$ the angle between the bonds $j$ and $k$. It is known that $\gamma_{j}=\alpha_{j}{ }^{\prime \prime}-\alpha_{j}{ }^{\perp}$; where $\alpha_{\| /}$and $\alpha_{\perp}$ are, respectively, the principal optical polarizabilities parallel and perpendicular to the bond considered.

If the molecules under study are in an anisotropic solvent, which is almost always the case, the depolarized intensity* scattered by the solution, $i_{\text {solution, }}$ is the sum of the depolarized intensities scattered by the solute in the presence of solvent, $i_{\text {solute }}$, and by the solvent in the presence of solute, $i_{\text {solvent }}$. Therefore, in Lorentz's internal field approximation it can be $\operatorname{seen}^{8}$ that for unpolarized light

$$
\begin{aligned}
i_{\text {solute }} & =i_{\text {solution }}-i_{\text {solvent }} \\
& =\frac{16 \pi^{4}}{135 \lambda^{4}} \operatorname{pr}^{2}\left(n^{2}+2\right)^{2} V E^{2}
\end{aligned}
$$

If the molecules studied are flexible chains existing in several different conformations, the mean value $\left\langle\gamma^{2}\right\rangle$ must be considered. In eq 3 $\lambda$ is the wavelength of the incident light beam in vacuo (here $5460 \AA$ ), $p$ the number of anisotropic molecules per $\mathrm{cm}^{3}$ of solution, $n$ the refractive index of the solution, $V$ the scattering volume, and $E$ the amplitude of the incident electric field.

If one notices that $p=C \mathscr{S} / M$ where $C$ is the solute concentration in $\mathrm{g} \mathrm{cm}^{-3}, M$ its molecular weight and $\mathscr{N}$ the Avogadro number, eq 3 can be written as

$$
i_{\text {solute }}=\frac{16 \pi^{4}}{135 \lambda^{4}} \mathscr{N} C\left(\frac{\gamma^{2}}{M}\right)\left(n^{2}+2\right)^{2} V E^{2}
$$

In practice $^{6,7}$ the intensity measurements are carried out by comparison with a standard liquid, cyclohexane, for which has been determined at a wavelength of $5460 \AA$ a scattered depolarized intensity per unit volume, and unit of incident photometric illumination of $23.15 \times 10^{-8}$ $\mathrm{cm}^{-1}$. This last value has been obtained by utilizing for the corresponding Rayleigh ratio of benzene the value of $16.3 \times 10^{-6} \mathrm{~cm}^{-1}$.

Treatment of the Results

If there are no solute-solute and solute-

* Often referred to as $H_{\mathrm{v}}$. solvent interactions, the molecular optical anisotropy determined from eq 4 should be independent of the solute concentration. In fact, the above interactions sometimes cause a variation, with the concentration, of the molecular optical anisotropy which consequently is not perfectly constant in the concentration range under study. The formula is only rigorously exact near the origin for infinitely diluted solutions. In this case the solute-solute interactions become negligible and the interactions due to the solvent are those of the pure liquid. That is why it is necessary during each determination to check that the experimental function

$$
\frac{i_{\text {solute }}}{\left(n^{2}+2\right)^{2}}=f(C)
$$

varies proportionally to the solute concentration at constant scattering volume and photometric illumination. The determination of the slope at the origin leads to the optical anisotropy of a solute molecule surrounded by solvent molecules. This value should be dependent upon the solvent but the molecular optical anisotropies of various solutes with a similar chemical constitution in the same solvent will be comparable. 9,10

During the analysis of experimental results, it is also necessary to consider the influence of various factors specific to polymers, such as polydispersity of samples and the polymer sizes compared to the wavelength of the light used. With regard to the first point, eq 4 shows that if the solution is composed of different molecular species $i$ in $C_{i}$ concentration and if all the particles have approximately the same refractive index, which is generally close to reality, ${ }^{11}$ we have

$$
i_{\text {solute }}=K \sum_{i} C_{i} \frac{\gamma_{i}^{2}}{M_{i}}=K C\left\langle\frac{\gamma^{2}}{M}\right\rangle
$$

that is

$$
\left\langle\gamma^{2} / M\right\rangle=\frac{\sum_{i} C_{i}\left(\gamma_{i}{ }^{2} / M_{i}\right)}{\sum_{i} C_{i}}=\sum_{i} w\left(M_{i}\right)\left(\gamma_{i}{ }^{2} / M_{i}\right)
$$

where $w\left(M_{i}\right)$ is the weight distribution function. Eq 5 shows that the experiment gives the weightaverage value of $r^{2} / M$. If some polydispersity is present, the chain being however a Gaussian 


\section{G. FourCHE and M.-T. JACQ}

one (that is $\gamma_{i}{ }^{2} / M_{i}=$ constant for each species $i$ ), the experimental results can be interpreted uniquely by using the horizontal part of the curve, $\left\langle\gamma^{2} \mid M\right\rangle=f(M)$. When the chain has not reached an asymptotic behavior, or if that behavior does not exist, the curve $w\left(M_{i}\right)$ giving the weight distribution must be known to be able to analyze the results correctly. Therefore it can be seen that in general, and especially in the case of short or relatively stiff chains, precise information on the polydispersity of the compounds under study will be needed.

Since polymer chains can reach sizes comparable to the wavelength used, it becomes necessary to reestablish, in the calculation of the scattered depolarized intensity, the phase relations between vibrations scattered by the various oscillators of the molecule. However, if the chain is assumed to be sufficiently long, as discussed by Horn, ${ }^{3}$ the depolarized component does not depend on the angle between the transmitted and scattered beams and does not vary with the molecular weight. Indeed, in this case, the molecular chain is sufficiently supple to limit the correlations between the orientations of its various parts to short distances and thus make the effect of interference negligible.

\section{SAMPLES}

\section{Atactic Polystyrene}

We were supplied, from the Pressure Chemical Company, ${ }^{12}$ with atactic PS samples which cover a wide range of molecular weight, (see definition below), i.e., 560, 2100, 3500, 9600, 20400, 97300,191000 , and 498000. They were prepared by anionic polymerization. ${ }^{13}$ All these products are characterized by a very narrow molecular weight distribution. Thus, for fractions ranging from 560 to 191000 the ratio of weight to number-average molecular weights as reported by the supplier is $\bar{M}_{w} / \bar{M}_{n} \leqq 1.06-1.10$. For the fraction 498000 the ratio is approximately $\bar{M}_{w} / \bar{M}_{n} \leqq 1.20$. Taking the low polydispersity of the collection of samples into account, we have characterized them by a single molecular weight $\bar{M}=\left(\bar{M}_{w}+\bar{M}_{n}\right) / 2$. The infrared spectra of these molecules as given by the supplier do not present any characteristic absorptions by carbonyl groups. It is known that styrene polymerizes easily and the linkage is always "head to tail." Therefore the polymer is linear and in general little branched. Its solubility is good

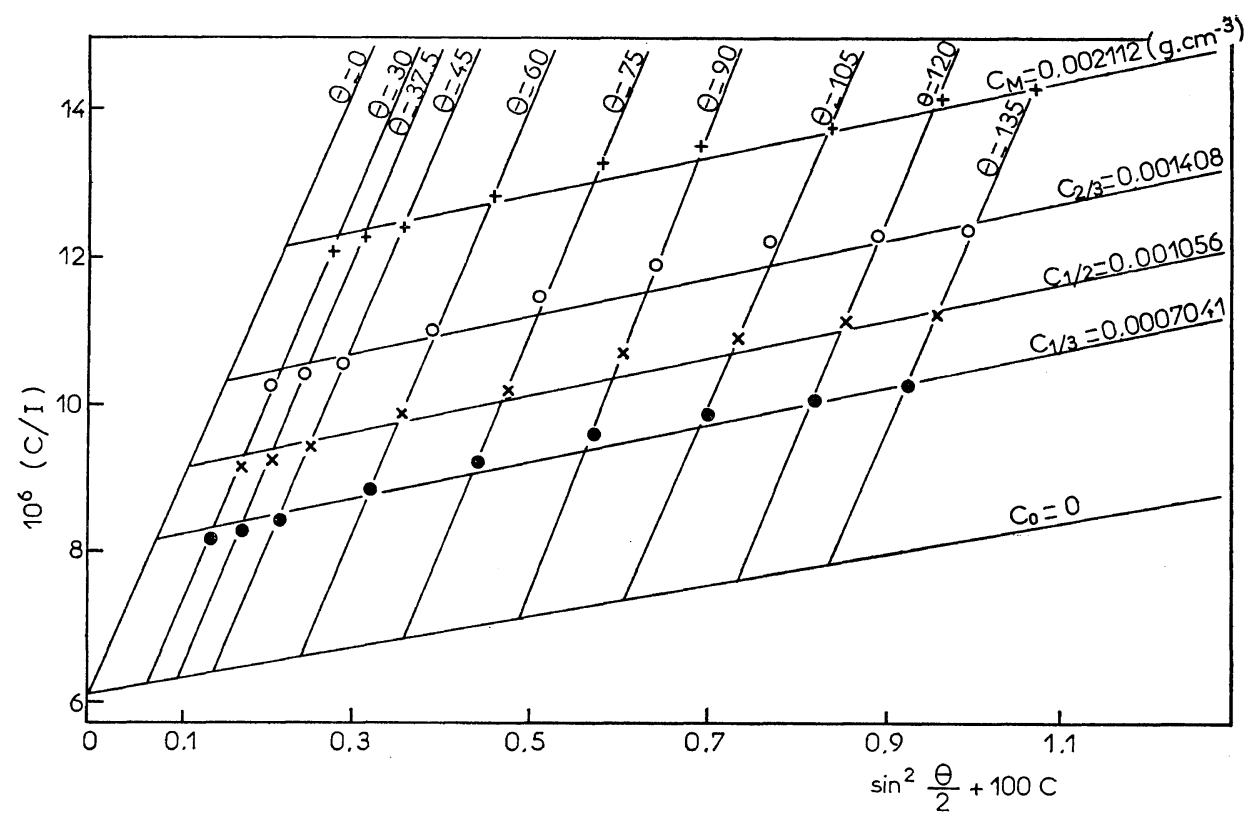

Figure 2. Zimm plot of fraction 498000 of atactic polystyrene in benzene solution. 
at room temperature in a great number of organic solvents. Thus, all these properties led us to choose this polymer as a model substance for a first study of depolarized Rayleigh scattering of long chains.

In order to be able to control all the factors which could be considered in the interpretation of our experimental results we have studied in particular the angular dissymetry of the fractions 97300, 191000, and 498000. In fact, it will be an experimental verification that, as predicted by the theory, ${ }^{3}$ the measurement of the depolarized component is not affected by interference phenomena when the size of the particles is comparable to the wavelength used. We have made, therefore, the conventional light scattering measurements with the use of a Fica apparatus $(\lambda=5460 \AA)$, and obtained the Zimm plots of these compounds in benzene solutions at $25^{\circ} \mathrm{C}$ (see example in Figure 2).

The results show that within the experimental accuracy there was no angular dependency for the fractions 97300 and 191000. In effect, we have obtained the correction factor $P^{-1}(90)$ for these two fractions was $1.02-1.04$ and 1.04 1.05 , respectively. On the contrary, the fraction 498000 revealed a strong angular dependency since $P^{-1}(90) \cong 1.18$. All these results were reobtained in another way by studying the intrinsic dissymmetry coefficient $[Z]=\left(I_{45} / I_{135}\right)_{C=0}$ and then by using the tables ${ }^{14}$ of constants
$P^{1}(90)=f([Z])$ for monodisperse random coils.

\section{Isotactic Polystyrene}

The isotactic polystyrene ${ }^{15}$ prepared with the usual Ziegler-Natta catalysts $\left[\mathrm{AlEt}_{3}-\mathrm{TiCl}_{3}\right.$ (or $\left.\mathrm{TiCl}_{4}\right)$ ] present a infrared spectrum typical of isotactic polymers. In order to obtain information concerning the polydispersity of the product, we determined the number (osmometer 502, mechrolab, membranes schleicher and schuell) and weight (Fica apparatus) average molecular weight. Measurements were made at $25^{\circ} \mathrm{C}$ in benzene solutions of concentrations approximately $10^{-3} \mathrm{~g} \mathrm{~cm}^{-3}$ for osmometry and $2 \times 10^{-4}$ $\mathrm{g} \mathrm{cm}^{-3}$ for light scattering, on three fractions $A$, $B$, and $C$ prepared ${ }^{15}$ according to the method of fractionation in tacticity described by Utiyama. ${ }^{20}$ Their number-average molecular weights $\bar{M}_{n}$ were 160000,185000 , and 240000 , respectively. An example of the results obtained by osmometry is given in Figure 3. For the first two fractions, we also estimated ${ }^{16}$ the viscosimetric average molecular weight $\bar{M}_{v}$ from intrinsic viscosity data obtained in tetralin ${ }^{17}$ at $100^{\circ} \mathrm{C}$ using the equation

$$
[\eta]=0.94 \times 10^{-4} \bar{M}_{v}^{0.73}
$$

The experimental values of $[\eta]$ in $\mathrm{d} l \mathrm{~g}^{-1}$ for $\mathrm{A}$ and $\mathrm{B}$ compounds are 0.915 and 1.1 , respectively which give $\bar{M}_{v}=294000$ for A and $\bar{M}_{v}=355000$ for $\mathbf{B}$.

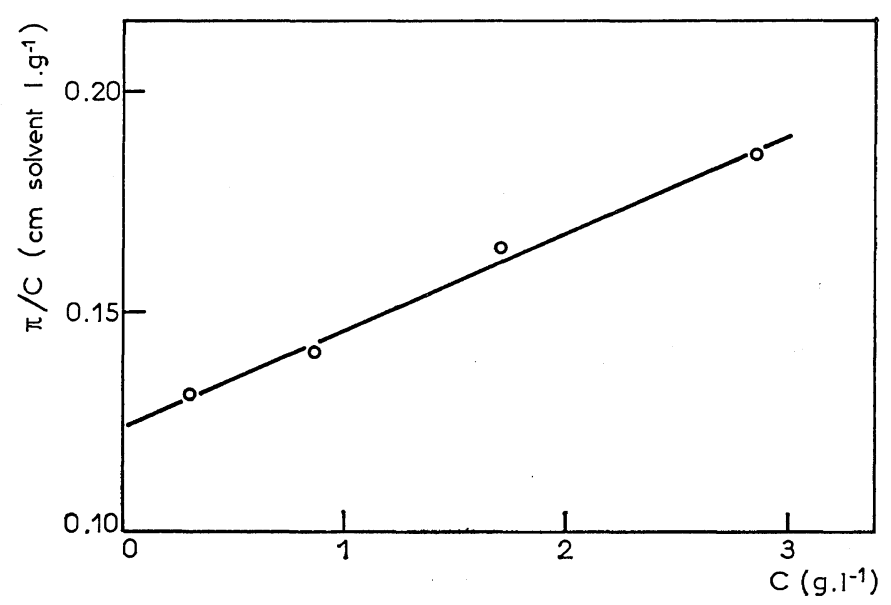

Figure 3. Osmotic data of sample $\mathrm{C}$ of isotactic polystyrene in benzene solution. 
The measurements of light scattering have presented some difficulties owing to the low solubility of isotactic polystyrene at room temperature and the heterogeneity of the samples. They have been done only on fractions $\mathrm{A}$ and B. Taking into account the strong observed molecular optical anisotropy of isotactic PS (see the following paragraph), the factor of depolarization $\rho$ of $\mathrm{A}$ and $\mathrm{B}$ samples in unpolarized light has been measured. We have remarked that this factor showed practically no variation with the concentration within the experimental error and that it was always approximately 0.01 . Therefore, the introduction of $\rho$ in the formulae does not greatly modify the results. It should be noted that this small value of $\rho$ quite understandable if it is assumed that the chains are sufficiently long because in this case the factor of depolarization becomes ${ }^{11}$ inversely proportional to the number of links in the molecule and decreases toward zero. ${ }^{11,18}$

The weight-average molecular weights $\bar{M}_{w}$ obtained are $\bar{M}_{w}=430000$ for A and $\bar{M}_{w}=700000$ for B. This leads to the ratio $\bar{M}_{w} / \bar{M}_{n}$ of 2.7 and 3.8 for A and B samples, respectively. We can reasonably assume that the polydispersity of the sample $C$ prepared under the same operative conditions is approximately of the same order of magnitude. The distribution of molecular weights of these samples is very broad.
However, these results are consistent with those reported in the literature. ${ }^{19,20}$

All the compounds studied revealed a large angular dissymmetry.

\section{DISSOLVING OF THE SAMPLES, CLARIFICATION}

This presents no difficulty at the room temperature for the atactic polystyrene in the solvents used (benzene, toluene, chloroform, decalin and cyclohexane) for our depolarized Rayleigh scattering measurements. On the other hand the dissolution of the isotactic polystyrenes cannot be made without difficulty. Therefore, at the concentrations reached in benzene or toluene for isotactic polystyrene we were unable to make measurements of depolarized Rayleigh scattering because the anisotropic component of the pure solvent was too high compared with that of the solute. We were then led to use, as did Reiss, ${ }^{21}$ 1,1,2,2-tetrachloroethane (TCE) and tetrahydrofuranne (THF), whose depolarized Rayleigh scattering in much weaker.

The removal of dust and small particles of extraneous matter from the solutions was carried out by filtering them through fine sintered glass of low porosity (mean diameter of the pores 1 to $2 \mu$ ). The absence of extraneous particles in the solution was checked by illuminating the

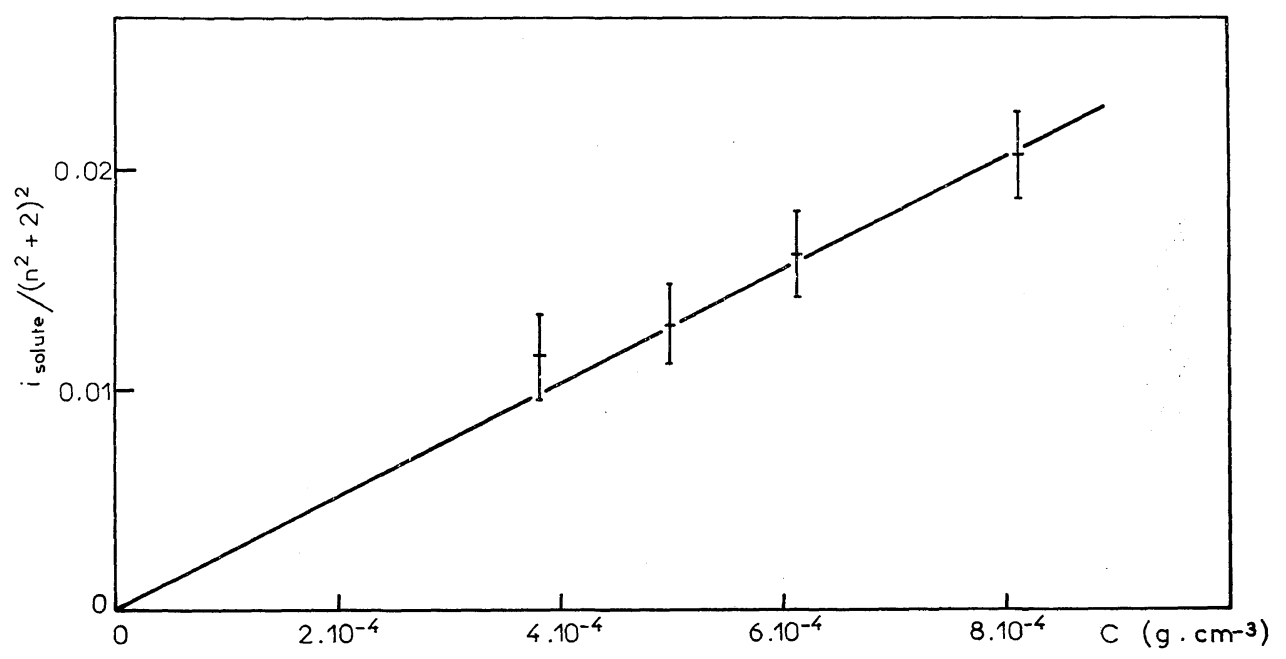

Figure 4. Concentration dependence of the scattered depolarized intensity for sample B of isotactic polystyrene in TCE solution. 
solution with the high intensity light of an arc, and by viewing it through a magnifying lens.

We have verified within the limit of experimental error that the experimental function $i_{\text {solute }} /\left(n^{2}+2\right)^{2}=f(C)$ was proportional to the concentration (see Figure 4), especially for all samples of isotactic PS studied. This linearity indicates in particular a complete dissolving of the polymer in the solution. In fact, it was not possible for isotactic polymers to control the concentrations by the usual method of refractive index because its variation was extremely small. On one hand this was due to the low solubilities used and on the other hand, in the case of TCE, to the fact that its refractive index was very close to that of the polymer. It is known that isotactic PS in solution has a tendency to form solid aggregates slowly; this phenomenon occurs more quickly if the concentration is higher. However, at the maximum concentration, around $8 \times 10^{-4} \mathrm{~g} \mathrm{~cm}^{-3}$, used for depolarized Rayleigh scattering measurements in TCE and THF, the presence of molecular aggregates is most unlikely, as previously noted. ${ }^{21}$ Since the amount of product was small, it was not possible to make a new determination of the molecular weight of those polymers by light scattering in other solvents (in particular TCE and THF) with thermodynamic properties appreciably different from that of benzene. It can indeed be assumed $^{20}$ that, if the molecular weight remains constant, the solution is in a molecularly dispersed state. We shall return to this question in the next paragraph.

\section{EXPERIMENTAL RESULTS AND DISCUSSION}

We have summarized the ensemble of experimental values of the $\left\langle\gamma^{2} / M\right\rangle$ ratios at $25^{\circ} \mathrm{C}$ for atactic and isotactic PS in Tables I and II, respectively. The experimental accuracy of about $5 \%$ for atactic polymers is noticeably higher in the case of isotactic chains due to the measurement difficulties. We have also listed in Table I the experimental optical anisotropies of meso and racemic 2,4-diphenylpentane (DPP) determined in cyclohexane and trimethyl 1,1,3cyclohexane, and, for comparison in our interpretation (see part II of this series), that of ethylbenzene in cyclohexane. The accuracy for these last compounds is about 2 or $3 \%$.

In order to examine experimentally the effect of polydispersity on the molecular optical anisotropy of polymers, we have given in Table III some results obtained by mixing in chloroform, in equal weights, two different fractions (noted in parentheses in column 1 of the Table) of atactic PS. The weight- and number-average molecular weights of these samples have been calculated by considering that the atactic PS fractions used were monodisperse. For the

Table I. Experimental values at $25^{\circ} \mathrm{C}$ of $\left\langle\gamma^{2} / M\right\rangle$ ratios for atactic polystyrenes, diphenylpentanes and ethylbenzene in various solvents

\begin{tabular}{lcccccc}
\hline Fractions & $\begin{array}{c}\text { Benzene } \\
(1.50198)^{\mathrm{a}}\end{array}$ & $\begin{array}{c}\text { Toluene } \\
(1.49773)\end{array}$ & $\begin{array}{c}\text { Chloroform } \\
(1.44385)\end{array}$ & $\begin{array}{c}\text { Decalin } \\
(1.47808)\end{array}$ & $\begin{array}{c}\text { Cyclo } \\
(1.42560)\end{array}$ & $\begin{array}{c}\text { 1,1,3-Tri- } \\
\text { methyl cyclo }\end{array}$ \\
\hline 560 & 0.45 & & 0.53 & 0.54 & 0.55 & \\
2100 & 0.72 & & 0.71 & 0.69 & 0.70 & \\
3500 & 0.70 & 0.75 & 0.71 & 0.71 & 0.70 & \\
9600 & 0.78 & 0.79 & 0.76 & 0.74 & 0.77 & \\
20400 & 0.77 & 0.85 & 0.82 & 0.87 & & \\
97300 & 0.75 & 0.84 & 0.88 & & & \\
191000 & 0.83 & 0.86 & & & 0.621 & 0.607 \\
498000 & 0.76 & 0.90 & & & 0.590 & 0.580 \\
meso-DPP & & & & & 0.572 & \\
Racem DPP & & & & & & \\
Ethylbenzene & & & & & & \\
\hline
\end{tabular}

a The refractive indices of the solvents at $25^{\circ} \mathrm{C}$ for $\lambda=5460 \AA$ have been written in parentheses. 


\section{G. Fourche and M.-T. JACQ}

convenience of the later discussion, we have also shown the $\left\langle\gamma^{2} \mid M\right\rangle$ values found in the chloroform for each of the monodisperse fractions together with the variations in $\%$ between those last values and these related to the polydisperse product.

The shape of the curves, $\left\langle\gamma^{2} / M\right\rangle v s$. $M$, obtained in different solvent with atactic and isotactic PS is shown in Figure 5. The $\left\langle\gamma^{2} / M\right\rangle$ of the isotactic chains are quite clearly larger than those of the atactic chains. Thus, for

Table II. Experimental values at $25^{\circ} \mathrm{C}$ of $\left\langle\gamma^{2} / M\right\rangle$ ratios for isotactic polystyrenes in various solvents

\begin{tabular}{ccl}
\hline \multicolumn{3}{c}{$\left\langle\gamma^{2} / M\right\rangle, \AA^{6} \mathrm{~g}^{-1}$} \\
\hline Fractions & TCE & THF \\
\hline A $\left(\bar{M}_{n}=160000\right)$ & 9.1 & 10 \\
B $\left(\bar{M}_{n}=185000\right)$ & 8.5 & 10.1 \\
C $\left(\bar{M}_{n}=240000\right)$ & 9.2 & \\
\hline
\end{tabular}

isotactic PS, the average of the results obtained for different samples in TCE and THF solutions, assuming that the chains are sufficiently long and are Gaussian in the range of molecular weights studied, is approximately $\left\langle\gamma^{2} \mid M\right\rangle_{\infty, \exp } \cong$ $9.3 \pm 1\left(\AA^{6} \mathrm{~g}^{-1}\right)$. On the other hand, for atactic

Table III. Comparison of experimental values at $25^{\circ} \mathrm{C}$ of $\left\langle\gamma^{2} / M\right\rangle$ ratios for polya and monodisperse chains of atactic polystyrene in chloroform

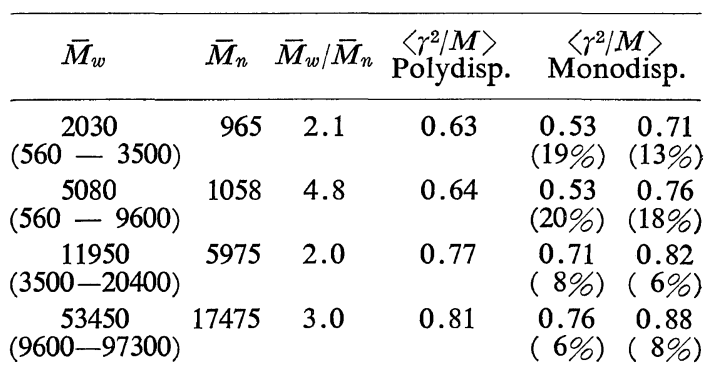

a Mixture in equal weights of the two fractions mentioned in parentheses in column 1 .

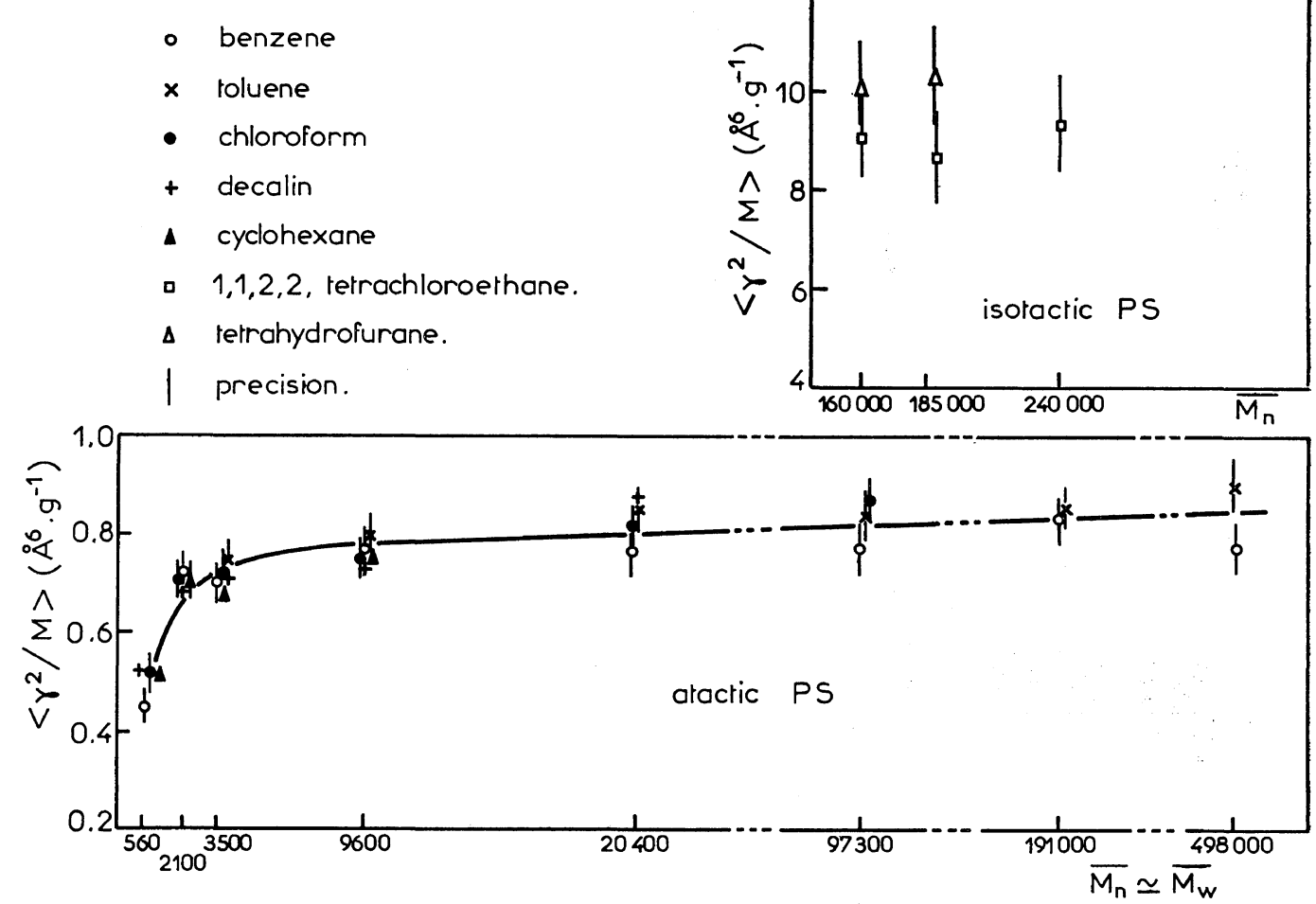

Figure 5. Experimental values of $\left\langle\gamma^{2} \mid \boldsymbol{M}\right\rangle$ ratios of atactic and isotactic polystyrene chains in various solvents at $25^{\circ} \mathrm{C}$. 
PS the average of the results found in several solvents gives $\left\langle r^{2} / M\right\rangle_{\infty, \exp } \cong 0.85_{ \pm} 0.05\left(\AA^{6} \mathrm{~g}^{-1}\right)$ as the limiting value. Consequently, the comparison of experimental optical anisotropies of atactic and isotactic chains reveals a considerable difference, approximately 10 fold, between the measured values.

Let us remark here that by light scattering Utiyama $^{20}$ has also observed for atactic and isotactic structures of PS enormous variations (40 to 60 fold) between the corresponding values of an anisotropy parameter related to the optical segment of the chain. Tsvetkov, et al., ${ }^{1}$ have also observed in flow birefringence studies that isotactic chains of polystyrene (PS), polypropylene (PP), poly(methyl methacrylate (PMM), and poly(butyl methacrylate) (PBM) are characterized by an anisotropy of the "statistical segment" very different from that of atactic molecules.

However, the results of optical anisotropy concerning these last two series of work have not been quantitatively interpreted in terms of the molecular conformation of the chain as a whole.

Except for the physical properties we have just discussed, most of the molecular quantities used up to now in trying to characterize the stereoregularity of polymers in solution have proved to be rather insensitive to this effect. We have compiled in Table IV a certain number of results concerning the optical anisotropies $\langle\Delta \alpha\rangle$ of the "statistical segment" (flow birefringence), the unpertubed mean chain dimensions $\left\langle R^{2}\right\rangle_{0}$ (viscosity, light scattering), and the mean square dipole moments $\left\langle\mu^{2}\right\rangle$ of some atactic and isotactic macromolecules. For each of those properties we have calculated the ratio of observed limiting values for the corresponding isotactic and atactic chains. It should be noted that it is necessary to be under theta conditions to study the mean dimensions of the chains, because the chain dimensions are dependent the long range interactions. On the other hand, neither the optical anisotropy, as we shall see later on, nor the dipole moment ${ }^{22}$ is very sensitive to this perturbation. We can see from Table IV that in general the effect of stereoregularity on molecular optical anisotropy $\left\langle\gamma^{2}\right\rangle$ and the anisotropy $\langle\Delta \alpha\rangle$ of the "statistical segment" is much more important than that observed, between 10 and $20 \%$ usually, in the study of unperturbed dimensions and dipole moments. With regard to this last point, it is necessary to point out that the $50-\%$ increase found by Krigbaum and $\mathrm{Roig}^{26}$ on the mean square dipole moment of isotactic PS compared to the atactic chain may not be very significant because of the low accuracy of the measurements on the isotactic compounds given by these authors. Let us mention, lastly, that the analysis of flow birefringence measurements from which the anisotropy $\langle\Delta \alpha\rangle$ is determined, still appears to be a most delicate process. This phenomenon involves both orientational and optical parameters which can only be separated at the cost of many hypotheses.

It seems, therefore, from these results, that the measurements of molecular optical anisotropy (for which the interpretation is much simpler)

Table IV. Influence of stereoregularity on the molecular optical anisotropy, anisotropy ${ }^{\mathrm{a}}$ of "statistical segment," mean dimensions, and dipole moments of some macromolecules ${ }^{b}$

\begin{tabular}{lcccc}
\hline Compounds & $\begin{array}{c}\left\langle\gamma^{2} / n\right\rangle_{\infty} \text { iso } \\
\left\langle\gamma^{2} / n\right\rangle_{\infty} \text { atac } \\
\text { (This work) }\end{array}$ & $\begin{array}{c}\frac{\langle\Delta \alpha\rangle_{\infty} \text { iso }}{\langle\Delta \alpha\rangle_{\infty} \text { atac }} \\
\left.\text { (Tsvetkov, et al. }{ }^{1}\right)\end{array}$ & $\begin{array}{c}\frac{\left(\left\langle R^{2}\right\rangle_{0} / n l^{2}\right)_{\infty} \text { iso }}{\left(\left\langle R^{2}\right\rangle_{0} / n l^{2}\right)_{\infty} \text { atac }} \\
(\text { Literature })\end{array}$ & $\begin{array}{c}\frac{\left(\left\langle\mu^{2}\right\rangle / n m^{2}\right)_{\infty} \text { iso }}{\left(\left\langle\mu^{2}\right\rangle / n m^{2}\right)_{\infty} \text { atac }} \\
(\text { Literature })\end{array}$ \\
\hline $\begin{array}{l}\text { PS } \\
\text { PP }\end{array}$ & 10.9 & 1.53 & $1.11^{23}$ & $1.08^{18}$ \\
PMM & 12.5 & $(1.10-1.20)^{24}$ & $(1.13-1.23)^{27}$ \\
PBM & 0.142 & $1.22^{25}$ & $\sim 1.10^{28}$
\end{tabular}

a Experimentally, $\langle\Delta \alpha\rangle$ is obtained ${ }^{1}$ and not the square of this quantity.

b $n$ is the number of bonds, $l^{2}$ and $m^{2}$ have their usual significance. ${ }^{18}$ 


\section{G. FourCHE and M.-T. JACQ}

of stereoregular polymers is an useful method for studying their degree of tacticity. This property appears in effect as an intrinsic quantity directly related to the local arrangement of substituents around successive asymmetric carbons.

Let us now examine in detail the shape of the curve in Figure 5. We can see that for the atactic polymer the asymptotic behavior is reached for a melecular weight of about 3000 , that is approximately 30 monomer units, and that the limiting value obtained is essentially the same, within experimental error, in benzene, toluene, and chloroform. In cyclohexane and decalin, we also find the same behavior. Therefore there does not seem to be any influence of chain interactions with the solvent in relation to the character of this one. In addition the effect of the intermolecular internal field, which under Lorentz's approximation is only a function of the refractive index of the media, doesn't seem to have any measurable influence on the anisotropy of polarizability of the chain studied. Thus, for fractions ranging from 560 to 9600 , the values of $\left\langle\gamma^{2} \mid M\right\rangle$ measured in cyclohexane $\left(n_{5460}^{25} \sim 1.42\right)$ and benzene $\left(n_{5460}^{25} \sim 1.50\right)$, solvents with very different refractive index, are in fact practically identical (see Table I). In order to minimize the effect of the internal field it would be interesting to carry out the measurements in solvents with refractive index as close as possible to that of the polymer $(\partial n / \partial c \cong 0)$. However, it is difficult to find any good solvents of the polymer studied which have at the same time a high refractive index (here $n \sim 1.60$ ) and a low molecular optical anisotropy. This last condition is necessary to have sufficient accuracy in the measurement of the intensity scattered by the solute. With regard to cyclohexane and decalin, we have not continued our measurements above molecular weight of 9600 and 20400 respectively because at these fractions we have observed a sudden increase of $\left\langle\gamma^{2} \mid M\right\rangle$ much larger than the experimental accuracy. We have attributed this effect to the possible formation of aggregates. This cause is indeed very likely since cyclohexane and decalin are, from the thermodynamic point of view, poor solvents ${ }^{21}$ for PS. For atactic PS, we can see on the curve of Figure 5 that the experimental points in benzene and toluene relative to fraction 498000, which present an angular disymmetry, are situated at 0.76 and $0.90\left(\AA^{6} \mathrm{~g}^{-1}\right)$, respectively. The precision of these last measurements is not as good as for the other compounds, most likely because of the greater difficulty in the transparency of the solutions for these large molecular weights. It would seem, nevertheless, in the absence of more information that, as predicted by Horn, ${ }^{3}$ the depolarized component of scattered light, $i_{\text {solute }} / C\left(n^{2}+2\right)^{2}$, which is directly proportional to $\left\langle\gamma^{2} \mid M\right\rangle$ is independent of the sizes of the chain when it is Gaussian. This occurs in practice when the chain has sufficient length.

We should also note that recent theoretical predictions $^{29}$ have shown that the excluded volume effect on molecular optical anisotropy of chain molecules should be extremely small compared to other properties (mean dimensions and radius of gyration) of the polymers. In fact we can see in Table I, by comparing the results for cyclohexane and decalin with those obtained in other solvents, that the variations at equal molecular weight of $\left\langle\gamma^{2} / M\right\rangle$ with the type of solvent rarely become larger than the experimental error. This equality can be easily understood only if the excluded volume is negligible in all these solvents.

The ensemble of previous results shows, finally, that we can hope to determine the molecular optical anisotropy of polymers with a good precision, even in the case of chains with large molecular weights dissolved in solvents with refractive index far enough from that of the polymer (see Table I). (That is to say under experimental conditions where the scattered intensity due to the isotropic component $\left(V_{\mathrm{v}}\right)$ has a relatively important value.)

An examination of the data in Table III, where we compare the $\left\langle\gamma^{2} / M\right\rangle$ ratios of poly and monodisperse chains of atactic PS in chloroform solution, shows that the polydispersity has a noticeable influence on $\left\langle\gamma^{2} \mid M\right\rangle$ when, in the mixture of studied chains, all of them have not reached an asymptotic behavior. Indeed, it can be seen that the $\left\langle\gamma^{2} / M\right\rangle$ ratios of very polydisperse nongaussian compounds (see lines 2 and 3 of the table) deviate rather sharply, 
Depolarized Rayleigh Scattering. I.

between 13 and $20 \%$, from the values corresponding to monodisperse fractions in the mixture. On the contrary as can be anticipated from eq 5, when the chains become almost Gaussian, the influence of polydispersity on the measurement of $\left\langle\gamma^{2} \mid M\right\rangle$ decreases fairly distinctly (the differences from 6 to $8 \%$ being much smaller) and should disappear when we arrive in the asymptotic domain.

For the isotactic polymer, we remark on Figure 5 that the values of $\left\langle\gamma^{2} / M\right\rangle$ in TCE and THF are essentially of the same order of magnitude, which excludes a posteriori the disturbing presence of aggregates in the solution.

\section{CONCLUSION}

The above work has pointed out the interest presented by the study of molecular optical anisotropy in the investigation of the stereoregularity of polymers and their local structure. The optical anisotropy appears indeed as an intrinsic molecular property not influenced by secondary effects (excluded volume); its determination by depolarized Rayleigh scattering can be done with an accuracy quite comparable to that obtained with other techniques of studying short distance interactions.

\section{REFERENCES}

1. V. N. Tsvetkov, J. Polym. Sci., 57, 727 (1962). V. N. Tsvetkov, S. Ya. Magarik, N. N. Boitsova, and M. G. Okuneva, ibid., 54, 635 (1961).

2. H. Benoit, Makromol. Chem., 18-19, 397 (1956), C.R. Acad. Sci., 236, 687 (1953).

3. P. Horn, Ann. Phys., 10, 386 (1955).

4. G. Weill, ibid., 6, 1063 (1961).

5. S. Krause and E. Cohn Ginsbert, Polymer, 3, 565 (1962).

6. C. Clement and P. Bothorel, J. Chim. Phys., 61, 878 (1964).

7. J. J. Piaud, ibid., 57, 215 (1962).

P. Bothorel, J. Colloid. Interfac. Sci., 27 (3), 529 (1968).

8. J. Cabannes, "La Diffusion de la Lumière," Presse Universitaire (1929), Paris.

9. P. Bothorel, Thesis Bordeaux (1958).
10. A. Rousset and R. Lochet, J. Phys. Radium, 13, 298 (1952).

11. H. Benoit and G. Weill, "Proceedings of the international Symposium on Macromolecular Chemistry," Prague, Sept. 1957, p 35-48.

12. 3419 Smallman St., Pittsburgh.

13. T. Altares, Jr., D. P. Wyman, and V. R. Allen, J. Polym. Sci., Part A, p 4533 (1964).

14. G. Champetier and L. Monnerie, "Introduction à la chimie macromoléculaire" Masson Ed. (1969), Paris.

15. Obtained by the courtesy of Professor Monnerie, Laboratoire de Chimie Macromoléculaire, Ecole Supérieure de Physique et Chimie, Paris.

16. A. Lety, private communication.

17. G. Natta, F. Danusso, and D. Sianesi, Makromol. Chem., 28, 253 (1958).

18. P. J. Flory, "Statistical mechanics of chain molecules," Interscience publishers, Inc., NewYork, N.Y. (1969).

19. K. Kawahara and R. Okada, J. Polym. Sci., 56, 163 (1962).

20. H. Utiyama, J. Phys. Chem., 69, $\mathrm{n}^{\circ} 12,4138$ (1965).

H. Utiyama and M. Kurata, Bull. Inst. Chem. Res. Kyoto Univ., 42, 128 (1964).

21. C. Reiss, J. Chim. Phys., 63, 1299-1327 (1966).

22. J. Marchal, C. Wippler, and H. Benoit, C.R. Acad. Sci., 241, 1266 (1955).

23. W. R. Krigbaum, D. K. Cappenter, and S. Newman, J. Phys. Chem., 62, 1586 (1959).

24. T. M. Birshtein and O. B. Ptitsyn, "Conformations of macromolecules," Interscience publishers Inc., New York, N.Y. (1966).

25. S. Krause and E. Cohn Ginsbert, J. Phys. Chem., 67, 1479 (1963).

26. W. R. Krigbaum and A. Roig, J. Chem. Phys., 31, 544 (1959).

27. H. A. Pohl, R. Backai, and W. P. Purcell, $J$. Phys. Chem., 64, 1701 (1960).

28. T. A. Borisova, L. L. Burshtein, and G.P. Mikhailov, Vysokomol. Soedin, 4, 1479 (1962).

29. B. Lemaire and G. Fourche, C.R. Acad. Sci., 272, 1071 (1971).

30. G. D. Patterson and P. J. Flory, J.C.S. Faraday II, 68, 1098 (1972).

31. J. R. Lalanne, F. B. Martin, and P. Bothorel, J. Colloid Interfac. Sci., 39601 (1972).

32. P. Bothorel and G. Fourche, J.C.S. Faraday II, 69, 441 (1973). 